\title{
Some Identities of Multiplicative Coupled Fibonacci Sequences
}

\author{
Bijendra Singh \\ School of studies in \\ Mathematics, Vikram \\ University, Ujjain, India
}

\author{
Shikha Bhatnagar \\ School of studies in \\ Mathematics, Vikram \\ University, Ujjain, India
}

\author{
Omprakash Sikhwal \\ Department of Mathematics, \\ Mandsaur Institute of \\ Technology, Mandsaur, India
}

\begin{abstract}
In the recent years, there has been much interest in development of coupled Fibonacci sequences. The concept of coupled Fibonacci sequences was first introduced by Atanassov, K. T. in 1985. He deliberated multiplicative coupled Fibonacci sequences of second order in 1995. Multiplicative coupled Fibonacci sequences are less known. In this paper we present some identities of multiplicative coupled Fibonacci sequences of second order under three specific schemes.
\end{abstract}

\section{Mathematics Subject Classification: 11B39, 11B37}

Keywords: Fibonacci sequence, Coupled Fibonacci Sequences, Multiplicative Coupled Fibonacci Sequences

\section{INTRODUCTION}

The concept of additive coupled Fibonacci sequence was first introduced by Atanassov, K. T. [1] in 1985. He defined four different schemes of additive coupled Fibonacci sequences [1] and called them 2-Fibonacci sequence (or 2-F sequences).

In 1995, Atanassov, K. T. [2] deliberated multiplicative coupled Fibonacci sequences of second order. He notified four different schemes of multiplicative coupled Fibonacci sequences.

Let $\left\{\alpha_{i}\right\}_{i=0}^{\infty}$ and $\left\{\beta_{i}\right\}_{i=0}^{\infty}$ be two infinite sequences with initial values $a, b, c$ and $d$. Then four different schemes of multiplicative coupled Fibonacci sequences of second order [2] are defined as follows:

$\begin{array}{lll}\text { First Scheme } & \alpha_{n+2}=\beta_{n+1} \cdot \beta_{n}, & n \geq 0 \\ & \beta_{n+2}=\alpha_{n+1} \cdot \alpha_{n}, & n \geq 0 . \\ & & \\ \text { Second Scheme } & \alpha_{n+2}=\alpha_{n+1} \cdot \beta_{n}, & n \geq 0 \\ & \beta_{n+2}=\beta_{n+1} \cdot \alpha_{n}, & n \geq 0 . \\ & & \\ \text { Third Scheme } & \alpha_{n+2}=\beta_{n+1} \cdot \alpha_{n}, & n \geq 0 \\ & \beta_{n+2}=\alpha_{n+1} \cdot \beta_{n}, & n \geq 0 . \\ \text { Forth Scheme } & \alpha_{n+2}=\alpha_{n+1} \cdot \alpha_{n}, & n \geq 0 \\ & \beta_{n+2}=\beta_{n+1} \cdot \beta_{n}, & n \geq 0 .\end{array}$

Singh B. and Sikhwal [5] have studied some fundamental properties for scheme (1.1). Rathore, G. P. S., Jain, S. and Sikhwal, O. [4], presents multiplicative coupled Fibonacci sequences of third order under two specific schemes. Sikhwal, O. [6], presented some fundamental properties of coupled Fibonacci sequences of higher order and multiplicative coupled Fibonacci sequences of second order.

In this paper, some identities of multiplicative coupled Fibonacci sequences of second order are presented under various schemes.

\section{MAIN RESULTS}

In this section, identities under scheme (1.2), (1.3) and (1.4) will be described.

Some identities of scheme (1.2) are discussed below:

Theorem (2.1). If $n \geq 0$ is any integer, then
(a). $\beta_{0} \cdot \alpha_{6 n}=\alpha_{0} \cdot \beta_{6 n}$,
(b). $\quad \beta_{1} \cdot \alpha_{6 n+1}=\alpha_{1} \cdot \beta_{6 n+1}$,
(c). $\beta_{2} \cdot \alpha_{6 n+2}=\alpha_{2} \cdot \beta_{6 n+2}$,
(d). $\quad \alpha_{0} \cdot \alpha_{6 n+3}=\beta_{0} \cdot \beta_{6 n+3}$,
(e). $\alpha_{1} \cdot \alpha_{6 n+4}=\beta_{1} \cdot \beta_{6 n+4}$,
(f). $\quad \alpha_{2} \cdot \alpha_{6 n+5}=\beta_{2} \cdot \beta_{6 n+5}$.

Proof. Induction method will be used to derive the identities.

(a) If $n=0$, then $\beta_{0} \cdot \alpha_{0}=\alpha_{0} \cdot \beta_{0}$.

Thus the result is true for $n=0$.

Now assume that the result is true for some integer $n \geq 1$. Then,

$$
\begin{aligned}
& \beta_{0} \cdot \alpha_{6 n+6}=\beta_{0}\left(\alpha_{6 n+5} \cdot \beta_{6 n+4}\right) \quad \text { (By scheme 1.2) } \\
& =\beta_{0}\left(\alpha_{6 n+4} \cdot \beta_{6 n+3}\right) \beta_{6 n+4} \\
& \text { (By scheme 1.2) } \\
& =\beta_{0} \cdot \alpha_{6 n+4}\left(\beta_{6 n+2} \cdot \alpha_{6 n+1}\right) \beta_{6 n+4} \\
& \text { (By scheme 1.2) } \\
& =\beta_{0} \cdot \alpha_{6 n+4}\left(\beta_{6 n+1} \cdot \alpha_{6 n}\right) \alpha_{6 n+1} \cdot \beta_{6 n+4} \\
& \text { (By scheme 1.2) } \\
& =\alpha_{6 n+4} \cdot \beta_{6 n+1}\left(\beta_{0} \cdot \alpha_{6 n}\right) \alpha_{6 n+1} \cdot \beta_{6 n+4} \\
& \text { (By scheme 1.2) } \\
& =\alpha_{6 n+4} \cdot \beta_{6 n+1}\left(\alpha_{0} \cdot \beta_{6 n}\right) \alpha_{6 n+1} \cdot \beta_{6 n+4} \\
& \text { (By hypothesis) } \\
& =\alpha_{0} \cdot \alpha_{6 n+4} \cdot \beta_{6 n+1} \cdot \alpha_{6 n+2} \cdot \beta_{6 n+4} \\
& =\alpha_{0} \cdot \beta_{6 n+6}
\end{aligned}
$$

Hence the result is true for all integers $n \geq 0$. 
Similar proofs can be given for remaining parts (b) to (f).

Theorem (2.2). If $n \geq 0$ is an integer, then

(a). $\alpha_{n+4}=\alpha_{n+2} \beta_{n+1}^{2} \alpha_{n}$,

(b). $\beta_{n+4}=\beta_{n+2} \alpha_{n+1}^{2} \beta_{n}$.

Theorem (2.3). If $n \geq 0$ is an integer, then $\alpha_{n+2} \beta_{n+2}=\left(\alpha_{0} \beta_{0}\right)^{F_{n+1}} \cdot\left(\alpha_{1} \beta_{1}\right)^{F_{n+2}}$.

Theorem (2.4). If $n \geq 0$ is an integer, then

(a). $\alpha_{n} \beta_{n+1} \alpha_{n+2}=\left(\alpha_{0} \beta_{0}\right)^{F_{n+1}} \cdot\left(\alpha_{1} \beta_{1}\right)^{F_{n+2}}$,

(b). $\beta_{n} \alpha_{n+1} \beta_{n+2}=\left(\alpha_{0} \beta_{0}\right)^{F_{n+1}} \cdot\left(\alpha_{1} \beta_{1}\right)^{F_{n+2}}$.

Theorem (2.5). If $n \geq 0$ is an integer, then

(a) $\frac{\alpha_{n+3}}{\beta_{n}}=\left(\alpha_{0} \beta_{0}\right)^{F_{n}} \cdot\left(\alpha_{1} \beta_{1}\right)^{F_{n+1}}$,

(b) $\frac{\beta_{n+3}}{\alpha_{n}}=\left(\alpha_{0} \beta_{0}\right)^{F_{n}} \cdot\left(\alpha_{1} \beta_{1}\right)^{F_{n+1}}$.

Theorem (2.6). If $n \geq 0$ is an integer, then $\prod_{k=0}^{n} \alpha_{k} \beta_{k}=\left(\alpha_{0} \beta_{0}\right)^{F_{n+1}} .\left(\alpha_{1} \beta_{1}\right)^{F_{n+2}-1}$.

Theorem (2.7). If $n \geq 0$ is an integer, then

(a). $\quad \alpha_{n+2}=\alpha_{1} \prod_{k=0}^{n} \beta_{k}$,

(b). $\quad \beta_{n+2}=\beta_{1} \prod_{k=0}^{n} \alpha_{k}$.

Theorem (2.8). If $n \geq 0$ is an integer, then
(a). $\frac{\prod_{k=0}^{6 n} \alpha_{k}}{\prod_{k=0}^{6 n} \beta_{k}}=\frac{\alpha_{0}}{\beta_{0}}$,
(b). $\frac{\prod_{k=0}^{6 n+1} \alpha_{k}}{\prod_{k=0}^{6 n+1} \beta_{k}}=\frac{\alpha_{0} \alpha_{1}}{\beta_{0} \beta_{1}}$,
(c). $\frac{\prod_{k=0}^{6 n+2} \alpha_{k}}{\prod_{k=0}^{6 n+2} \beta_{k}}=\frac{\alpha_{1}^{2}}{\beta_{1}^{2}}$,
(d). $\frac{\prod_{k=0}^{6 n+3} \alpha_{k}}{\prod_{k=0}^{6 n+3} \beta_{k}}=\frac{\alpha_{1}^{2}}{\beta_{1}^{2}} \frac{\beta_{0}}{\alpha_{0}}$,
(e). $\frac{\prod_{k=0}^{6 n+4} \alpha_{k}}{\prod_{k=0}^{6 n+4} \beta_{k}}=\frac{\alpha_{1} \beta_{0}}{\beta_{1} \alpha_{0}}$,
(f). $\frac{\prod_{k=0}^{n} \alpha_{k}}{\prod_{k=0}^{n} \beta_{k}}=1$.

Some identities of scheme (1.3) are discussed below:

Theorem (2.9). If $n \geq 0$ is an integer, then
(a). $\quad \alpha_{3 n+3}=\alpha_{3 n+1} \beta_{3 n+2}$,

(b). $\quad \beta_{3 n+3}=\beta_{3 n+1} \alpha_{3 n+2}$.

Theorem (2.10). If $n \geq 0$ is an integer, then

(a). $\alpha_{n+4}=\alpha_{n+2} \beta_{n+1}^{2} \alpha_{n}$,

(b). $\beta_{n+4}=\beta_{n+2} \alpha_{n+1}^{2} \beta_{n}$.

Theorem (2.11). If $n \geq 0$ is an integer, then $\alpha_{n+2} \beta_{n+2}=\left(\alpha_{0} \beta_{0}\right)^{F_{n+1}} \cdot\left(\alpha_{1} \beta_{1}\right)^{F_{n+2}}$.
Theorem (2.12). If $n \geq 0$ is an integer, then
(a). $\quad \alpha_{n} \beta_{n+1} \alpha_{n+2}=\left\{\begin{array}{l}\alpha_{0}^{2 F_{n+1}} \cdot \beta_{1}^{2 F_{n+2}}, n \text { is even } \\ \beta_{0}^{2 F_{n+1}} \cdot \alpha_{1}^{2 F_{n+2}}, n \text { is odd }\end{array}\right.$.
(b). $\quad \beta_{n} \alpha_{n+1} \beta_{n+2}=\left\{\begin{array}{l}\beta_{0}^{2 F_{n+1}} \cdot \alpha_{1}^{2 F_{n+2}}, n \text { is even } \\ \alpha_{0}^{2 F_{n+1}} \cdot \beta_{1}^{2 F_{n+2}}, n \text { is odd }\end{array}\right.$.

Theorem (2.13). If $n \geq 0$ is an integer, then (a) $\frac{\alpha_{n+3}}{\beta_{n}}=\left\{\begin{array}{l}\beta_{0}^{2 F_{n}} \cdot \alpha_{1}^{2 F_{n+1}}, n \text { is even } \\ \alpha_{0}^{2 F_{n}} \cdot \beta_{1}^{2 F_{n+1}}, n \text { is odd }\end{array}\right.$

(b) $\frac{\beta_{n+3}}{\alpha_{n}}=\left\{\begin{array}{l}\alpha_{0}^{2 F} \cdot \beta_{1}^{2 F_{n+1}}, n \text { is even } \\ \beta_{0}^{2 F_{n}} \cdot \alpha_{1}^{2 F_{n+1}}, n \text { is odd }\end{array}\right.$.

Theorem (2.14). If $n \geq 0$ is an integer, then $\prod_{k=0}^{n} \alpha_{k} \beta_{k}=\left(\alpha_{0} \beta_{0}\right)^{F_{n+1}} .\left(\alpha_{1} \beta_{1}\right)^{F_{n+2}-1}$.

Theorem (2.15). If $n \geq 0$ is an integer, then
(a) $\frac{\alpha_{3 n+5}}{\beta_{3 n+2}}=\left\{\begin{array}{l}\beta_{0}^{2 F_{3 n+2}} \cdot \alpha_{1}^{2 F_{3 n+3}}, n \text { is even } \\ \alpha_{0}^{2 F_{3 n+2}} \cdot \beta_{1}^{2 F_{3 n+3}}, n \text { is odd }\end{array}\right.$,
(b) $\frac{\beta_{3 n+5}}{\alpha_{3 n+2}}=\left\{\begin{array}{l}\alpha_{0}^{2 F_{3 n+2}} \cdot \beta_{1}^{2 F_{3 n+3}}, n \text { is even } \\ \beta_{0}^{2 F_{3 n+2}} \cdot \alpha_{1}^{2 F_{3 n+3}}, n \text { is odd }\end{array}\right.$,
(c) $\frac{\alpha_{3 n+6}}{\beta_{3 n+3}}=\left\{\begin{array}{l}\alpha_{0}^{2 F_{3 n+3}} \cdot \beta_{1}^{2 F_{3 n+4}}, n \text { is even } \\ \beta_{0}^{2 F_{3 n+3}} \cdot \alpha_{1}^{2 F_{3 n+4}}, n \text { is odd }\end{array}\right.$,
(d) $\frac{\beta_{3 n+6}}{\alpha_{3 n+3}}=\left\{\begin{array}{l}\beta_{0}^{2 F_{3 n+3}} \cdot \alpha_{1}^{2 F_{3 n+4}}, n \text { is even } \\ \alpha_{0}^{2 F_{3 n+3}} \cdot \beta_{1}^{2 F_{3 n+4}}, n \text { is odd }\end{array}\right.$,
(e) $\frac{\alpha_{3 n+7}}{\beta_{3 n+4}}=\left\{\begin{array}{l}\beta_{0}^{2 F_{3 n+4}} \cdot \alpha_{1}^{2 F_{3 n+5}}, n \text { is even } \\ \alpha_{0}^{2 F_{3 n+4}} \cdot \beta_{1}^{2 F_{3 n+5}}, n \text { is odd }\end{array}\right.$,
(f) $\frac{\beta_{3 n+7}}{\alpha_{3 n+4}}=\left\{\begin{array}{l}\alpha_{0}^{2 F_{3 n+4}} \cdot \beta_{1}^{2 F_{3 n+5}}, n \text { is even } \\ \beta_{0}^{2 F_{3 n+4}} \cdot \alpha_{1}^{2 F_{3 n+5}}, n \text { is odd }\end{array}\right.$

Finally, identities of scheme (1.4) are stated:

Theorem (2.16). If $n \geq 0$ is an integer, then (a). $\alpha_{n+4}=\alpha_{n+2} \alpha_{n+1}^{2} \alpha_{n}$,

(b). $\beta_{n+4}=\beta_{n+2} \beta_{n+1}^{2} \beta_{n}$.

Theorem (2.17). If $n \geq 0$ is an integer, then $\alpha_{n+2} \beta_{n+2}=\left(\alpha_{0} \beta_{0}\right)^{F_{n+1}} .\left(\alpha_{1} \beta_{1}\right)^{F_{n+2}}$.

Theorem (2.18). If $n \geq 0$ is an integer, then (a). $\alpha_{n} \alpha_{n+1} \alpha_{n+2}=\alpha_{0}^{2 F_{n+1}} \cdot \alpha_{1}^{2 F_{n+2}}$,

(b). $\beta_{n} \beta_{n+1} \beta_{n+2}=\beta_{0}^{2 F_{n+1}} \cdot \beta_{1}^{2 F_{n+2}}$.

Theorem (2.19). If $n \geq 0$ is an integer, then (a) $\frac{\alpha_{n+3}}{\alpha_{n}}=\alpha_{0}^{2 F_{n}} \cdot \alpha_{1}^{2 F_{n+1}}$, 
(b) $\frac{\beta_{n+3}}{\beta_{n}}=\beta_{0}^{2 F_{n}} \cdot \beta_{1}^{2 F_{n+1}}$.

Theorem (2.20). If $n \geq 0$ is an integer, then

(a) $\prod_{k=0}^{n} \alpha_{k}=\alpha_{0}^{F_{n+1}} \cdot \alpha_{1}^{F_{n+2}-1}$,

(b) $\prod_{k=0}^{n} \beta_{k}=\beta_{0}^{F_{n+1}} \cdot \beta_{1}^{F_{n+2}-1}$.

Theorem (2.21). If $n \geq 0$ is an integer, then
( a ) $\alpha_{3 n+3}=\alpha_{1} \prod_{k=0}^{3 n+1} \alpha_{k}$,
(b) $\beta_{3 n+3}=\beta_{1} \prod_{k=0}^{3 n+1} \beta_{k}$,
(c) $\alpha_{3 n+4}=\alpha_{1} \prod_{k=0}^{3 n+2} \alpha_{k}$,
(d) $\beta_{3 n+4}=\beta_{1} \prod_{k=0}^{3 n+2} \beta_{k}$,
(e) $\alpha_{3 n+5}=\alpha \prod_{k=0}^{3 n+3} \alpha_{k}$,
(f) $\beta_{3 n+5}=\beta_{1} \prod_{k=0}^{3 n+3} \beta_{k}$.

Theorem (2.22). If $n \geq 0$ is an integer, then
(a) $\frac{\alpha_{3 n+5}}{\alpha_{3 n+2}}=\alpha_{0}^{2 F_{3 n+2}} \cdot \alpha_{1}^{2 F_{3 n+3}}$,
(b) $\frac{\beta_{3 n+5}}{\beta_{3 n+2}}=\beta_{0}^{2 F_{3 n+2}} \cdot \beta_{1}^{2 F_{3 n+3}}$,
(c) $\frac{\alpha_{3 n+6}}{\alpha_{3 n+6}}=\alpha_{0}^{2 F_{3 n+3}} \cdot \alpha_{1}^{2 F_{3 n+4}}$,
(d) $\frac{\beta_{3 n+6}}{\beta_{3 n+3}}=\beta_{0}^{2 F_{3 n+3}} \cdot \beta_{1}^{2 F_{3 n+4}}$,
(e) $\frac{\alpha_{3 n+7}}{\alpha_{3 n+4}}=\alpha_{0}^{2 F_{3 n+4}} \cdot \alpha_{1}^{2 F_{3 n+5}}$,
(f) $\frac{\beta_{3 n+7}}{\beta_{3 n+4}}=\beta_{0}^{2 F_{3 n+4}} \cdot \beta_{1}^{2 F_{3 n+5}}$.

The proof of all above identities under schemes 1.2 to 1.4 can be given by induction method.

\section{CONCLUSION}

This paper describes identities of multiplicative coupled Fibonacci sequences of second order under various schemes. Many similar identities can be developed for higher order multiplicative coupled Fibonacci sequences.

\section{ACKNOWLEDGMENTS}

Authors are thankful to reviewers for their valuable comments in improvement of manuscript.

\section{REFERENCES}

[1] Atanassov, K. T., Atanassov, L. C. and Sasselov, D. D., 1985. A New Perspective to the Generalization of the Fibonacci Sequence, The Fibonacci Quarterly, Vol. 23, No. 1, 21-28.

[2] Atanassov, K. T., 1995. Remark on a New Direction for a Generalization of the Fibonacci Sequence, The Fibonacci Quarterly, Vol. 33, No. 3, 249-250.

[3] Atanassov, K T., Atanassov V., Shannon A.and Turner J., 2002. New Visual Perspective On Fibonacci Number, World Scientific Publishing Company, Singapore.

[4] Rathore, G. P. S., Jain, S. and Sikhwal, O., 2012. Multiplicative Coupled Fibonacci Sequences of Third Order, International Journal of Contemporary Mathematical Sciences, Vol. 7, No. 31, 1535 - 1540.

[5] Singh, B. and Sikhwal, O., 2010. Multiplicative Coupled Fibonacci Sequences and Some Fundamental Properties, International Journal of Contemporary Mathematical Sciences, Vol. 5, No. 5, 223-230.

[6] Sikhwal, O. 2012. Generalization of Fibonacci sequence: An Intriguing Sequence, Lap Lambert Academic Publishing GmbH \& Co. KG, Germany. 